\title{
Tenofovir and Entecavir Have Similar Renal Adverse Events on Hepatocel- Iular Carcinoma Patients Treated with Transarterial Chemoembolization
}

\author{
Young Youn $\mathrm{Cho}^{1,2^{*}}$, Young Hwan Choi ${ }^{2^{*}}$, Su Jong Yu', Eun Ju Cho', Jeong-Hoon Lee', Yoon Jun Kim¹, Jung-Hwan Yoon \\ 'Department of Internal Medicine and Liver Research Institute, Seoul National University College of Medicine, Seoul; ${ }^{2}$ Department of \\ Internal Medicine, Chung-Ang University Hospital, Seoul, Korea
}

Received Jan. 31, 2019

Revised Feb. 21, 2019

Accepted Feb. 25, 2019
Background/Aims: Tenofovir disoproxil fumarate (TDF) is potentially nephrotoxic in chronic hepatitis B patients. Hepatocellular carcinoma (HCC) patients treated using transarterial chemoembolization (TACE) are at an increased risk of renal injury. The aim of this study was to determine whether TDF is associated with more renal adverse events than entecavir (ETV) in HCC patients treated with TACE.

Methods: In this retrospective single-center study, we selected 53 HCC patients who were treated with TDF from January 2012 to July 2013 and had their first TACE procedure in the same period. These patients were matched by age and sex to patients treated with ETV.

Results: There were no significant differences in baseline characteristics, including HCC factors, and nephrotoxic drug use, between the two groups. The median follow-up period was 17.0 and 20.0 months for the TDF and ETV groups, respectively. There was no difference during the follow-up period between the TDF and ETV groups in the increase in creatinine over $0.5 \mathrm{mg} / \mathrm{dL}(17.0 \%$ and $17.0 \%, P=1.00$, respectively) and the decrease in eGFR over $25 \%$ (43.4\% and $41.5 \%, P=0.84$, respectively). Multivariate analysis revealed that Child-Pugh class over $B$ (hazard ratio $[\mathrm{HR}], 7.30 ; 95 \%$ confidence interval $[\mathrm{Cl}] 2.79-19.10 ; P<0.01$ ) was associated with increase in creatinine, and Child-Pugh class over B (HR, 82.74; 95\% Cl 12.31-555.83; $P<0.01$ ) and Barcelona-Clinic Liver Cancer stage over B (HR, 14.93; 95\% Cl 1.60-139.51; $P=0.02)$ were associated with decrease in eGFR.

Conclusions: TDF has comparable safety to that of ETV for HCC patients undergoing TACE. (J Liver Cancer 2019;19:128-135)

Keywords: Hepatocelluar carcinoma; Transarterial chemoembolization; Hepatitis B, chronic; Kidney diseases; Antiviral agents

\footnotetext{
Corresponding author : Su Jong Yu

Department of Internal Medicine and Liver Research Institute, Seoul National University Hospital, Seoul National University College of Medicine, 103 Daehak-ro, Jongno-gu, Seoul 03080, Korea

Tel. +82-2-2072-2228, Fax. +82-2-743-6701

E-mail; ydoctor2@hanmail.net

https://orcid.org/0000-0001-8888-7977

*Both authors contributed equally to this work.
}

\section{INTRODUCTION}

Copyright $\odot 2019$ by The Korean Liver Cancer Association. All rights reserved.

This is an Open Access article distributed under the terms of the Creative Commons Attribution Non-Commercial License (http://creativecommons.org/licenses/by-nc/4.0/) which permits unrestricted non-commercial use, distribution, and reproduction in any medium, provided the original work is properly cited. 
cers in the preclinical setting. ${ }^{3}$ However, there is no evidence of increased incidence of cancer in humans. TDF is a more potent drug compared to ETV, showing superior viral suppression and lower incidence of drug resistance. However, there is concern regarding nephrotoxicity of TDF. ${ }^{4}$ Previous studies assumed the mechanism of nephrotoxicity was mitochondrial damage, causing direct damage of the proximal tubule. ${ }^{5}$ In addition, there were several cases of Fanconi syndrome after TDF treatment. ${ }^{5-7}$

Transarterial chemoembolization (TACE) is recommended for advanced HCC patients in the Barcelona-Clinic Liver Cancer (BCLC) B stage in most guidelines. ${ }^{8}$ However, in real-world practice, physicians utilize TACE beyond the guidelines, and TACE is the most frequently used treatment strategy for HCC. ${ }^{9}$ HCC patients undergoing TACE are at an increased risk of kidney injury. The risk factors for acute kidney injury after TACE are poor liver function, hypertension, decreased serum albumin levels, and high dose of radiocontrast in TACE treatments. ${ }^{10}$ These patients are also frequently exposed to contrast computed tomography (CT) scans and use nephrotic drugs such as diuretics.

There has been no study comparing the risk of renal events by antiviral treatments in HCC patients undergoing TACE treatment. Therefore, the results of this study can give evidence when choosing the antiviral agent for HCC patients. The aim of our study is to compare the renal toxicity of TDF versus ETV in HCC patients undergoing TACE treatment.

\section{METHODS}

\section{Patient selection}

A total of 771 HCC patients with CHB treated with TDF between January 2012 and July 2013 at the Seoul National University Hospital were screened for this study. The inclusion criteria were: 1) age $>18$ years, 2) CHB patients initially treated with TDF without changing the treatment method, 3) HCC patients undergoing TACE as their first treatment for HCC, and 4) baseline estimated glomerular filtration rate (eGFR) $>30 \mathrm{~mL} / \mathrm{min} / 1.73 \mathrm{~m}^{2}$ and not receiving hemodialysis. The exclusion criteria were: 1 ) history of prescription of other antiviral agents for CHB treatment and 2) other malignancy requiring treatment. Fifty-three patients were eventually included in this study. A total of 2,076 patients treated with ETV monotherapy between January 2012 and July 2013 with the same inclusion/exclusion criteria were screened and 53 randomly selected patients were matched by age and sex (the TDF versus the ETV groups, Fig. 1). The study protocol was approved by the Institutional Review Board of Seoul National University Hospital, which follows the ethical guidelines of the World Medical Association Declaration of Helsinki.

\section{Selection of antiviral therapy}

The indication for antiviral therapy followed the guidelines of the Korean Association for the Study of the Liver, which is similar to the global guidelines. ${ }^{11}$

\section{Assessment of renal function}

The eGFR was calculated using the Modification of Diet in Renal Disease Study equation. ${ }^{12}$ Serial measurements of serum creatinine (Cr) and eGFR were performed per each patient's needs. Changes in renal function were measured retrospectively. The primary outcome was nephrotoxicity, which was defined as: 1) a decrease in eGFR by over $25 \%$ from the baseline,
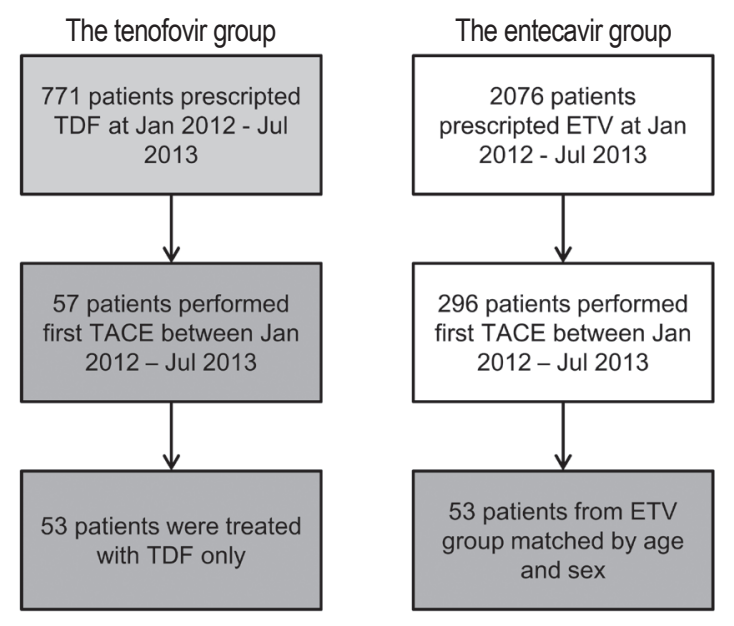

Figure 1. Patient selection. TDF, tenofovir; ETV, entecavir; TACE, transarterial chemoembolization. 
and 2) an increase in Cr by over $0.5 \mathrm{mg} / \mathrm{dL}$ from the baseline.

\section{Statistical analysis}

To compare baseline characteristics, we used the $t$-test for continuous variables and the chi-square test for non-continuous variables. The renal events in the TDF and ETV groups were compared using the chi-square test, and for estimation of time factors, the Kaplan-Meier curve with log-rank test for comparison was used. Prognostic factors were evaluated using univariate and multivariate analysis performed using the Cox proportional hazards model. All statistical analyses were performed using SPSS Statistics, version 22.0 (IBM Corp., Armonk, NY, USA), and results were considered statistically significant at a two-sided $P<0.05$.

Table 1. Baseline characteristics

\begin{tabular}{|c|c|c|c|}
\hline & Tenofovir group $(n=53)$ & Entecavir group $(n=53)$ & $P$-value* \\
\hline Age (years) & $56.9 \pm 8.9$ & $56.8 \pm 9.0$ & 0.92 \\
\hline \multicolumn{4}{|l|}{ Sex } \\
\hline Male & $43(81.1)$ & $42(79.2)$ & 0.81 \\
\hline Child-Pugh score & & & 0.56 \\
\hline A & $40(75.5)$ & $43(81.1)$ & \\
\hline B & $10(18.9)$ & $9(17.0)$ & \\
\hline C & $3(5.7)$ & $1(1.9)$ & \\
\hline Diabetes mellitus & $8(15.1)$ & $6(11.3)$ & 0.57 \\
\hline Hypertension & $7(13.2)$ & $3(5.7)$ & 0.18 \\
\hline Serum creatinine (mg/dL) & $0.81 \pm 0.16$ & $0.83 \pm 0.2$ & 0.58 \\
\hline eGFR-MDRD (mL/min/1.73 m²) & $98.7 \pm 22.2$ & $96.8 \pm 21.8$ & 0.65 \\
\hline eGFR under 60 & $0(0.0)$ & $3(5.7)$ & 0.24 \\
\hline \multicolumn{4}{|l|}{ Tumor factor } \\
\hline HCC size $(\mathrm{cm})$ & $4.8 \pm 4.5$ & $4.2 \pm 4.5$ & 0.51 \\
\hline Portal vein invasion & & & 0.88 \\
\hline Main & $6(11.4)$ & $4(7.5)$ & \\
\hline Major branch & $4(7.5)$ & $4(7.5)$ & \\
\hline Segmental & $5(9.4)$ & $4(7.5)$ & \\
\hline BCLC stage (A/B/C, \%) & $45.3 / 26.4 / 28.3$ & 49.1/17.0/34.0 & 0.49 \\
\hline \multicolumn{4}{|l|}{ Renal factor } \\
\hline Diuretics treatment & $20(37.7)$ & $21(39.6)$ & 0.65 \\
\hline Maximum spironolactone dose (25 mg) & $4.0 \pm 2.0$ & $4.3 \pm 1.8$ & 0.70 \\
\hline Maximum furosemide dose (40 mg) & $1.1 \pm 0.6$ & $1.2 \pm 0.9$ & 0.91 \\
\hline Other nephrotoxic drug use & $9(17.0)$ & $10(18.9)$ & 0.80 \\
\hline Adriamycin dose (mg) & $113.6 \pm 83.2$ & $120.7 \pm 124.5$ & 0.73 \\
\hline Cisplatin dose (mg) & $21.6 \pm 53.2$ & $32.8 \pm 64.9$ & 0.34 \\
\hline Number of TACE treatment (month) & $0.31 \pm 0.55$ & $0.22 \pm 0.20$ & 0.48 \\
\hline Number of contrast CT (month) & $0.47 \pm 0.66$ & $0.41 \pm 0.19$ & 0.27 \\
\hline
\end{tabular}

Values are presented as mean \pm standard deviation or number (\%) unless otherwise indicated.

eGFR, estimated glomerular filtration rate; MDRD, modification of diet in renal disease; TACE, transarterial chemoembolization; CT, computed tomography.

*Quantative variables by 2-sample t-test, qualitative variables by chi-square test. 


\section{RESULTS}

\section{Baseline characteristics}

We selected 53 patients treated with TDF from a single center using the inclusion/exclusion criteria (described in the patient selection section) and matched them with ETV-treated patients. Baseline characteristics were collected at the time of starting the TACE treatment. Patients were predominantly male $(81.0 \%)$ and the distribution of the Child-Pugh class was comparable between the TDF and ETV groups $(P=0.56$,

Table 1). The baseline tumor factors such as maximum tumor size, degree of portal vein thrombosis, BCLC stage, and the baseline kidney function between the TDF and ETV groups were well balanced (Table 1).

We investigated the use of nephrotoxic drugs throughout the follow up period, focusing on the use of diuretics and radiocontrast agents. Both the TDF and ETV groups had used comparable amount of diuretics, chemotherapeutic agents during TACE, and other nephrotoxic drugs such as non-steroidal anti-inflammatory drugs and angiotensin receptor blockers. The number of TACE treatments and the number of contrast CT scans per month were comparable between the two groups. Taken together, we can assume that the use of radiocontrast agents was also comparable between the two groups.

\section{Renal events of the TDF group compared to that of the ETV group}

The median duration of follow-up was 17.0 months in the TDF group (interquartile range [IQR], 9.0-22.0 months), and 20.0 months in the ETV group (IQR, 12.0-30.0 months). During the follow up period, there were no differences in renal events in the TDF and ETV groups: increase in Cr over $0.5 \mathrm{mg} / \mathrm{dL}(17.0 \%$ and $17.0 \%, P=1.00$, respectively, Table 2$)$;

Table 2. Changes in renal function during the follow-up period

\begin{tabular}{lccc}
\hline & Tenofovir group $(\mathbf{n}=\mathbf{5 3})$ & Entecavir group $(\mathbf{n = 5 3 )}$ & $\boldsymbol{P}^{\text {-value }}$ \\
\hline Increase in Cr over $0.5 \mathrm{mg} / \mathrm{dL}$ & $9(17.0)$ & $9(17.0)$ & 1.00 \\
Decrease in eGFR over 25\% & $23(43.4)$ & $22(41.5)$ & 0.84 \\
\hline
\end{tabular}

Values are presented as number (\%).

eGFR, estimated glomerular filtration rate; $\mathrm{Cr}$, serum creatinine.

"Chi-square test.
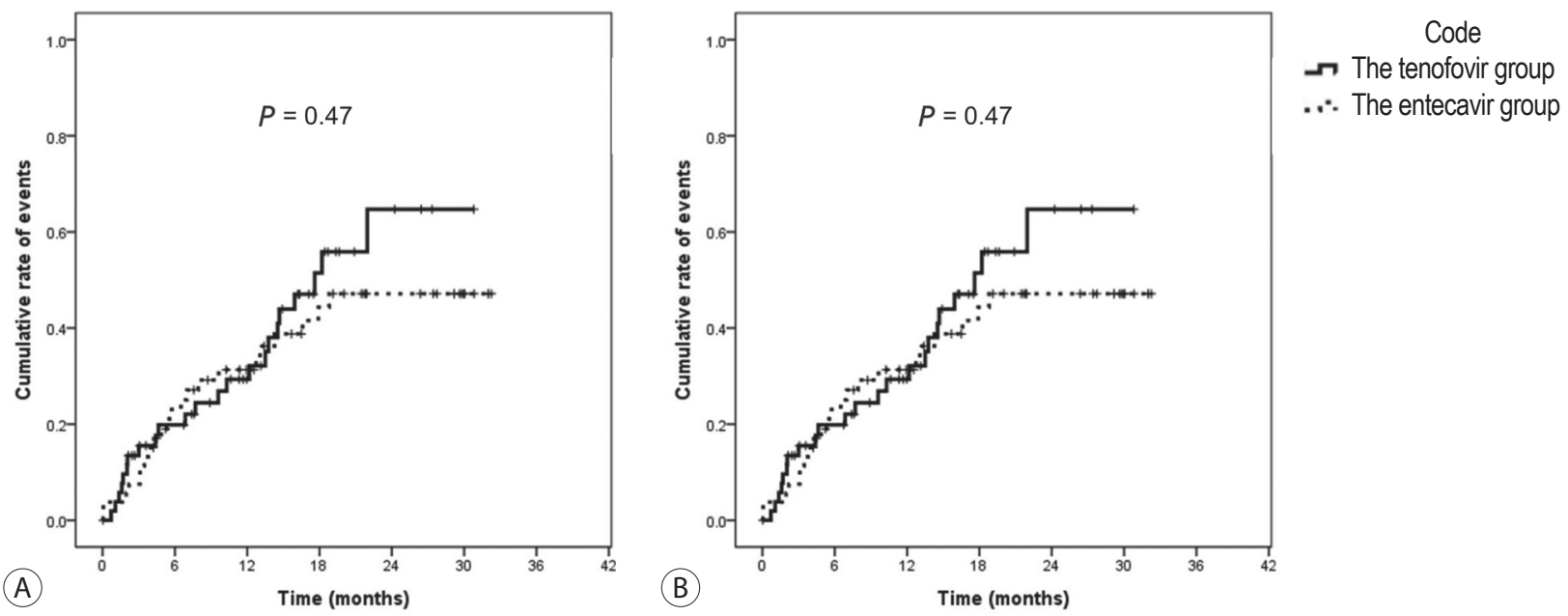

Figure 2. Renal events of the tenofovir group compared to those of the entecavir group. (A) Kaplan-Meier curve of increase in creatinine over 0.5 $\mathrm{mg} / \mathrm{dL}$. (B) Kaplan-Meier curve of decrease in eGFR over $25 \%$.

"Chi-square test. 
Journal of Liver Cancer

Volume 19 Number 2, September 2019

Table 3. Antiviral dose reduction due to acute renal failure

\begin{tabular}{lccc}
\hline & Tenofovir group $(\mathbf{n}=\mathbf{2 0})$ & Entecavir group (n=21) & $\boldsymbol{P}_{\text {-value }}$ \\
\hline Number of dose reduction cases & $4(19.0)$ & $3(15.0)$ & 1.00 \\
Standard dose (48 hours) & $1(4.8)$ & $0(0.0)$ & $2(10.0)$ \\
Standard dose (72 hours) & $2(9.5)$ & $1(5.0)$ & \\
Standard dose (week) & $1(4.8)$ & & \\
\hline
\end{tabular}

Values are presented as number (\%).

${ }^{*}$ Chi-square test.

Table 4. Risk factors of renal events

\begin{tabular}{|c|c|c|c|c|c|c|c|c|}
\hline \multirow{3}{*}{ Parameter } & \multicolumn{4}{|c|}{ eGFR decrease over $25 \%$} & \multicolumn{4}{|c|}{ Creatinine increase over $0.5 \mathrm{mg} / \mathrm{dL}$} \\
\hline & \multicolumn{2}{|c|}{ Univariate } & \multicolumn{2}{|c|}{ Multivariate } & \multicolumn{2}{|c|}{ Univariate } & \multicolumn{2}{|c|}{ Multivariate } \\
\hline & HR $(95 \% \mathrm{Cl})$ & $P$-value ${ }^{*}$ & $\mathrm{HR}(95 \% \mathrm{Cl})$ & $P$-value ${ }^{*}$ & $\mathrm{HR}(95 \% \mathrm{Cl})$ & $P$-value ${ }^{*}$ & $\mathrm{HR}(95 \% \mathrm{Cl})$ & $P$-value ${ }^{*}$ \\
\hline Age & $0.98(0.94-1.01)$ & 0.13 & & & $0.95(0.90-1.00)$ & 0.07 & & \\
\hline \multicolumn{9}{|l|}{ Sex } \\
\hline Female & $1.49(0.73-3.04)$ & 0.27 & & & $0.53(0.12-2.32)$ & 0.40 & & \\
\hline Diabetes & $1.92(0.92-4.00)$ & 0.08 & & & $1.22(0.35-4.21)$ & 0.76 & & \\
\hline Hypertension & $1.11(0.43-2.81)$ & 0.83 & & & $1.06(0.24-4.62)$ & 0.94 & & \\
\hline $\log (H B V D N A)$ & $1.02(0.84-1.23)$ & 0.88 & & & $1.06(0.79-1.43)$ & 0.69 & & \\
\hline \multicolumn{9}{|l|}{ Child Pugh Score } \\
\hline A & 1 & & 1 & & 1 & & 1 & \\
\hline$B-C$ & $4.81(2.61-8.86)$ & $<0.01^{*}$ & $7.30(2.79-19.10)$ & $<0.01^{*}$ & $13.75(5.10-37.08)$ & $<0.01^{*}$ & $82.74(12.31-555.83)$ & $<0.01^{*}$ \\
\hline Tumor size & $1.01(0.93-1.09)$ & 0.82 & & & $1.06(0.94-1.17)$ & 0.37 & & \\
\hline Tumor number & $1.51(1.20-1.91)$ & $<0.01^{*}$ & & & $2.28(1.60-3.26)$ & $<0.01^{*}$ & & \\
\hline \multicolumn{9}{|l|}{ Portal vein thrombosis } \\
\hline Main & $4.52(1.95-10.50)$ & $<0.01^{*}$ & & & $19.69(5.62-69.00)$ & $<0.01^{*}$ & & \\
\hline Major branch & $3.11(1.27-7.58)$ & $0.01^{*}$ & & & $14.38(4.12-50.13)$ & $<0.01^{*}$ & & \\
\hline Segmental branch & $1.96(0.75-5.12)$ & 0.17 & & & $5.32(1.27-22.25)$ & $0.02^{*}$ & & \\
\hline \multicolumn{9}{|l|}{ BCLC stage } \\
\hline A & 1 & & & & 1 & & & \\
\hline$B-C$ & $1.84(1.02-3.35)$ & $0.04^{*}$ & & & $11.38(2.60-49.72)$ & $<0.01^{*}$ & $14.93(1.60-139.51)$ & $0.02^{*}$ \\
\hline Diuretics treatment & $2.24(1.20-4.19)$ & $0.01^{*}$ & & & $4.15(1.64-10.50)$ & $<0.01^{*}$ & & \\
\hline Aldactone dose (tablet) & $1.36(1.03-1.80)$ & $0.03^{*}$ & & & $1.25(0.91-1.71)$ & 0.18 & & \\
\hline Furix dose (tablet) & $2.44(0.94-6.32)$ & 0.07 & & & $1.24(0.51-3.02)$ & 0.64 & & \\
\hline Number of TACE (months) & $2.97(1.15-7.63)$ & $0.02^{*}$ & & & $4.52(1.49-13.72)$ & $<0.01^{*}$ & & \\
\hline Adriamycin dose & $1.00(1.00-1.00)$ & 0.36 & & & $1.00(1.00-1.00)$ & 0.86 & & \\
\hline Cisplatin dose & $1.00(1.00-1.01)$ & 0.66 & & & $1.01(1.00-1.01)$ & 0.09 & & \\
\hline $\begin{array}{l}\text { Number of contrast CT } \\
\text { (months) }\end{array}$ & $1.81(0.62-5.23)$ & 0.27 & & & $2.09(0.79-5.58)$ & 0.14 & & \\
\hline Treatment of entecavir & $0.80(0.45-1.45)$ & 0.47 & & & $0.93(0.37-2.33)$ & 0.87 & & \\
\hline
\end{tabular}

Values are presented as number (range).

eGFR, estimated glomerular filtration rate; Cr, serum creatinine; HR, Hazard ratio; Cl, confidence interval; BCLC, Barcelona clinic liver cancer; TACE, transarterial chemoembolization; $\mathrm{CT}$, computed tomography.

${ }^{*}$ Cox proportional hazard regression, $P<0.05$. 
and decrease in eGFR under 25\% (43.4\% and 41.5\%, $P=0.84$, respectively, Table 2). Analysis of time effects using the log-rank method also revealed no difference in the increase in Cr over $0.5 \mathrm{mg} / \mathrm{dL}(P=0.87$, Fig. $2 \mathrm{~A})$, and decrease in eGFR under $25 \%(P=0.47$, Fig. $2 \mathrm{~B})$ in the TDF group compared to the ETV group. Dose reduction of diuretics was also comparable between the TDF and ETV groups (19.0\% and $15.0 \%, P=1.00$, respectively, Table 3 ).

\section{Predictors of renal events}

Cox regression analysis revealed that there was no difference in the risk of renal events in the ETV group compared to the TDF group, decrease in eGFR over 25\% (hazard ratio [HR], 0.80; 95\% confidential interval [CI], 0.45-1.45, $P=0.47$, Table 4), and increase in Cr over $0.5 \mathrm{mg} / \mathrm{dL}$ (HR, 0.93 ; $95 \% \mathrm{CI}, 0.37-2.33 ; P=0.87$, Table 4 ). In the multivariate Cox regression analysis, Child-Pugh score $\mathrm{B}-\mathrm{C}$ was the only factor associated with a decrease in eGFR over $25 \%$ (HR, 7.30; 95\% CI, 2.79-19.10), and Child-Pugh score B-C (HR, 82.74; 95\% CI, 12.31-555.83; $P<0.01$, Table 4) and BCLC stage B-C (HR, 14.93; $95 \%$ CI, 1.60-139.51; $P<0.01$, Table 4) were associated with an increase in Cr over $0.5 \mathrm{mg} / \mathrm{dL}$.

\section{Cause of renal events}

There was a higher frequency of decrease in eGFR over $25 \%(\mathrm{n}=45,42.5 \%)$ than of increase in Cr over $0.5 \mathrm{mg} / \mathrm{dL}$ $(\mathrm{n}=18,17.0 \%)$. Increase in Cr over $0.5 \mathrm{mg} / \mathrm{dL}$ was associated more with significant clinical events such as hepatic decompensation, HCC progression, infection, bleeding, and TACE (Table 5).

\section{DISCUSSION}

These results of $53 \mathrm{CHB}$ patients using TDF matched with ETV-treated patients revealed that the choice of antiviral treatment was not associated with significant renal events when HCC patients were undergoing TACE treatment. There is no previous study comparing the renal events between TDF and ETV with TACE treatments, therefore, we suggest the results of this study can aid clinical decisions when choosing antiviral drugs for CHB-related HCC patients.

There is controversy regarding renal adverse events associated with TDF and ETV in CHB patients. A previous study compared increase in $\mathrm{Cr}$ and decrease in eGFR in TDF-treated and ETV-treated CHB patients. The study concluded that the risk of nephrotoxicity was comparable between the two treatment options. ${ }^{4}$ However, another recent multi-center study composed of a large multi-center cohort of CHB patients $^{13}$ evaluated nephrotoxicity of TDF and found that TDF caused a decrease in eGFR, although the clinical significance was questionable. Another multi-center study conducted a long term follow-up of 49 months and concluded that there were no differences in renal adverse events between the TDF and ETV treatments. ${ }^{14}$

HCC patients undergoing TACE are at an increased risk of

Table 5. Cause of renal events

\begin{tabular}{lcc}
\hline & Decrease in eGFR over $\mathbf{2 5 \%}(\mathbf{n}=\mathbf{4 5})$ & Increase in Cr over $\mathbf{0 . 5} \mathbf{~ m g / d L ~}(\mathbf{n}=\mathbf{1 8})$ \\
\hline Hepatic decompensation/HCC progression & $5(11.1)$ & $8(44.4)$ \\
Hepatorenal syndrome & $2(4.4)$ & $2(11.1)$ \\
\hline Infection without shock & $5(11.1)$ & $3(16.7)$ \\
Septic shock & $3(6.7)$ & $2(11.1)$ \\
Bleeding & $1(2.2)$ & $1(5.6)$ \\
Post-operative volume depletion & $2(4.4)$ & $0(0.0)$ \\
\hline TACE & $2(4.4)$ & $1(5.6)$ \\
Unknown & $25(55.6)$ & $1(5.6)$ \\
\hline
\end{tabular}

Values are presented as number (\%).

eGFR, estimated glomerular filtration rate; Cr, serum creatinine; HCC, hepatocellular carcinoma; TACE, transarterial chemoembolization. 
kidney injury. Previous studies revealed that the risk factors for acute kidney injury (AKI) after TACE are poor liver function, poor renal function, and use of nephrotoxic agents..$^{10,15}$ As AKI in HCC patients undergoing TACE is related to higher mortality and permanent renal dysfunction, ${ }^{15}$ physicians must be aware of the use of nephrotoxic drugs when treating these patients. The results of our study can give evidence when choosing the antiviral agent for HCC patients.

Previous studies evaluating the renal toxicities of antiviral agents have shown that considerable time elapses before the occurrence of renal adverse events. One study in HIV patients revealed a long time gap from the initiation of the TDF treatment to significant renal adverse events. In that study, progression of significant nephrotoxicity occurred 4 to 5 years following TDF treatment. ${ }^{16}$ We assume that in our study, the duration of TDF exposure was not enough to cause renal adverse events in HCC patients undergoing TACE treatments, because the life expectancy of these patients is significantly shorter than that of $\mathrm{CHB}$ patients without HCC. Therefore, there was no difference in renal adverse events between the TDF and ETV groups. The HCC patients undergoing TACE are at an increased risk of AKI and the cause of kidney injury is variable as seen in Table 5 . As there was no risk of nephrotoxicity with TDF treatment, we can also suppose that TDF use does not cause synergetic kidney injury when HCC patients undergoing TACE exhibit these kinds of renal injuries.

The limitations of this study are the retrospective nature of the design, being a single center study, and the small sample size. To remove potential bias, we selected TDF or ETV monotherapy patients, and this exclusion criterion caused a substantial decrease in the study population. In addition, it was difficult to judge the cause of AKI in TACE-treated HCC patients, as these patients were exposed to multiple potential nephrotoxic treatments.

In conclusion, TDF has comparable safety to that of ETV for patients undergoing TACE. The results of our study can aid clinicians when choosing between TDF and ETV treatment in HCC patients. However, further long-term study needs to be performed.

\section{Conflicts of Interest}

The authors have no conflicts to disclose.

\section{REFERENCES}

1. Lee WM. Hepatitis B virus infection. N Engl J Med 1997;337:17331745.

2. Lok AS, McMahon BJ, Brown RS Jr, Wong JB, Ahmed AT, Farah $W$, et al. Antiviral therapy for chronic hepatitis B viral infection in adults: a systematic review and meta-analysis. Hepatology 2016;63:284-306.

3. Bristol-Myers Squibb. Archived drug label, [Internet]. United States: Bristol-Myers Squibb; [cited Jan 1 2019]. Available from: https://dailymed.nlm.nih.gov/dailymed/archives/fdaDrugInfo. cfm?archiveid $=10048$.

4. Gish RG, Clark MD, Kane SD, Shaw RE, Mangahas MF, Baqai S. Similar risk of renal events among patients treated with tenofovir or entecavir for chronic hepatitis B. Clin Gastroenterol Hepatol 2012;10:941-946.

5. Hall AM, Hendry BM, Nitsch D, Connolly JO. Tenofovir-associated kidney toxicity in HIV-infected patients: a review of the evidence. Am J Kidney Dis 2011;57:773-780.

6. Hwang HS, Park CW, Song MJ. Tenofovir-associated Fanconi syndrome and nephrotic syndrome in a patient with chronic hepatitis B monoinfection. Hepatology 2015;62:1318-1320.

7. Cho H, Cho Y, Cho EJ, Lee JH, Yu SJ, Oh KH, et al. Tenofovirassociated nephrotoxicity in patients with chronic hepatitis B: two cases. Clin Mol Hepatol 2016;22:286-291.

8. Yu SJ. A concise review of updated guidelines regarding the management of hepatocellular carcinoma around the world: 2010 2016. Clin Mol Hepatol 2016;22:7-17.

9. Park JW, Chen M, Colombo M, Roberts LR, Schwartz M, Chen PJ, et al. Global patterns of hepatocellular carcinoma management from diagnosis to death: the BRIDGE Study. Liver Int 2015;35:21552166.

10. Zhou C, Wang R, Ding Y, Du L, Hou C, Lu D, et al. Prognostic factors for acute kidney injury following transarterial chemoembolization in patients with hepatocellular carcinoma. Int J Clin Exp Pathol 2014;7:2579-2586.

11. Korean Association for the Study of the Liver. KASL clinical practice guidelines: management of chronic hepatitis B. Clin Mol Hepatol 2016;22:18-75.

12. Levey AS, Stevens LA, Schmid CH, Zhang YL, Castro AF 3rd, Feldman $\mathrm{HI}$, et al. A new equation to estimate glomerular filtration rate. Ann Intern Med 2009;150:604-612.

13. Koklu S, Gulsen MT, Tuna Y, Koklu H, Yuksel O, Demir M, et al. Differences in nephrotoxicity risk and renal effects among anti- 
viral therapies against hepatitis B. Aliment Pharmacol Ther 2015:41:310-319.

14. Riveiro-Barciela M, Tabernero D, Calleja JL, Lens S, Manzano ML, Rodriguez FG, et al. Effectiveness and safety of entecavir or tenofovir in a spanish cohort of chronic hepatitis b patients: validation of the page-b score to predict hepatocellular carcinoma. Dig Dis Sci 2017;62:784-793.

15. Hao JF, Zhang LW, Bai JX, Li YJ, Liu JN, Zhang XL, et al. Incidence, risk factors, and prognosis of acute kidney injury following transarterial chemoembolization in patients with hepatocellular carcinoma: a prospective cohort study. Indian J Cancer 2015;51 Suppl 2:e3-e8.

16. Monteagudo-Chu MO, Chang MH, Fung HB, Bräu N. Renal toxicity of long-term therapy with tenofovir in HIV-infected patients. J Pharm Pract 2012;25:552-559. 